\title{
Impact of Acquisition Wideband Noise on Synchrophasor Measurements: a Design Perspective
}

\author{
David Macii, Senior Member, IEEE, Daniele Fontanelli, Member, IEEE, Grazia Barchi, Member, IEEE, \\ Dario Petri, Fellow, IEEE \\ Dep. of Industrial Engineering - University of Trento, Trento, Italy, Email: david.macii@unitn.it \\ Institute for Renewable Energy - EURAC Research, Bolzano, Italy, Email: grazia.barchi@eurac.edu
}

\begin{abstract}
Next-generation Phasor Measurement Units (PMU) are expected to be more accurate than existing ones, especially to address the stricter requirements of future active distribution grids. From this perspective, the influence of acquisition wideband noise (which includes multiple contributions both in amplitude and in phase) has to be carefully evaluated. In this context, the contribution of this paper is twofold. First, it provides a general framework to evaluate the effect of wideband noise on synchrophasor amplitude, phase, frequency and rate of change of frequency (ROCOF) estimation. The results of this analysis show that the influence of wideband noise can become critical for compliance with the requirements reported in the IEEE Standards C37.118.1-2011 and C37.118.1a-2014, especially for frequency and ROCOF estimation. In addition, the paper reports general guidelines to choose the PMU effective resolution and sampling rate for which the impact of wideband noise on both $P$ Class and $M$ Class PMUs is negligible.
\end{abstract}

Keywords-Phasor measurement units, signal processing algorithms, additive white noise, estimation theory, uncertainty.

\section{INTRODUCTION}

In future distribution networks, the increasing diffusion of distributed energy resources (possibly equipped with energy storage) and the potential bidirectional power flows caused by large variations of load and generation profiles, will require high-accuracy instruments able to measure the amplitude, phase, frequency and rate of change of frequency (ROCOF) of electrical waveforms in real-time [1]. The so-called Phasor Measurement Units (PMUs) are expected to play a central role to this end [2]. Most of the algorithms proposed in the last few years are conceived not only to mitigate the effect of steadystate disturbances, but also to track dynamic oscillations in amplitude and in phase. While the techniques based on the Interpolated DFT (IpDFT) and its variants can compensate mainly the effect of static or quasi-static off-nominal frequency deviations [3]-[5], the dynamic models relying on the Taylor's series expansion of the synchrophasor can be successfully used to estimate its derivatives with respect to time, thus improving accuracy even in non-stationary or in transient conditions [6][8]. Moreover, synchrophasor, frequency and ROCOF can be estimated together, possibly using a single data record [9]-[13]. At the moment, the main reference documents to test and to compare PMU performances are the IEEE Standard C37.1182011 and the IEEE Amendment C37.118.1a-2014 [14]-[16]. However, the effect of wideband noise on PMU measurement accuracy is not included in the testing conditions, probably because it is implicitly supposed to be made negligible at the design level. As a consequence, the effect of wideband noise on PMU synchrophasor estimation algorithms has been analyzed just marginally in the scientific literature, either using simulations (e.g. to evaluate the performances of specific algorithms [17], [18]) or in experimental testbeds (e.g. for calibration purposes [19], [20]). However, in view of deploying the PMUs in distribution systems, the accuracy of phase, frequency and ROCOF measurements has to be considerably improved with respect to current standard limits [21]. Thus, the effect of noise can become relevant. For instance, the accuracy required for phasor angle measurements can be even smaller than $1 \mathrm{mrad}$ [22] and the impact of noise has to be reduced, as emphasized in [23], where an algorithm based on the DFT and two bandpass filters is proposed to this purpose. Moreover, since frequency and ROCOF are defined, respectively, as the first and the second derivative with respect to time of the waveform instantaneous phase, they are expected to be quite sensitive to wideband noise, although the actual impact of such noise on estimation accuracy depends of the adopted algorithm. Some PMU manufacturers have recently pointed out that quantization, thermal noise and other noise sources affecting the input section of a PMU can significantly affect measurement accuracy [24], [25]. Nevertheless, a theoretical analysis of the impact of noise on synchrophasor amplitude, phase, frequency and ROCOF estimation is basically missing in the literature. This paper contributes to fill this gap by extending the preliminary analysis presented in [26]. First, Section II shows how signal-to-noise ratio, estimation algorithm properties and data acquisition parameters affect measurement accuracy. In Section III some meaningful simulation results confirm the correctness of the theoretical analysis in different testing conditions. Then, Section IV provides general guidelines to keep the impact of noise under control for both $\mathrm{P}$ Class and $\mathrm{M}$ Class PMUs. Finally, Section V concludes the paper.

\section{THEORETICAL ANALYSIS}

\section{A. Waveform model}

As known, a PMU is conceived to measure the phasor, the frequency and the ROCOF of a voltage or a current waveform at a reference time $t_{r}$ synchronized to the UTC. To this aim, a PMU has to process the data collected within observation intervals of settable duration. In particular, each 
interval typically comprises approximately an integer number $C$ of waveform cycles at nominal frequency $f_{0}$ (equal to 50 $\mathrm{Hz}$ or $60 \mathrm{~Hz}$ ) [8], [14]. Let us assume that each observation interval includes $N$ samples. If $f_{s}$ is the sampling rate of the PMU and $M=\frac{f_{s}}{f_{0}}$ is the number of samples within a single nominal waveform period, then $N \cong C \cdot M$. Thus, the waveform collected by a PMU within the $r$-th observation interval (with $r$ integer) can be modeled as follows:

$$
x_{r}(n)=A_{r}(n) \cos \left[\theta_{r}(n)+\boldsymbol{\eta}_{p}(n)\right]+\varepsilon_{h_{r}}(n)+\boldsymbol{\eta}_{a}(n)
$$

where:

- $-\frac{N-1}{2} \leq n \leq \frac{N-1}{2}$ (for $N$ odd) or $-\frac{N}{2} \leq n \leq \frac{N}{2}-1$ (for $N$ even), since the reference time, at which synchrophasor, frequency and ROCOF are measured, lie exactly in the center of the $r$-th observation interval;

- $A_{r}(n)=A \cdot\left[1+\varepsilon_{a_{r}}(n)\right]$ is the waveform amplitude given by the superimposition of the mean value $A$ and the intrinsic oscillations described by $\varepsilon_{a_{r}}(n)$;

- $\theta_{r}(n)=\frac{2 \pi}{M}(1+\delta) n+\varepsilon_{p_{r}}(n)+\phi_{r}$ is the instantaneous phase of the waveform which depends on:

- the initial phase $\phi_{r}$ at reference time $t_{r}$;

- the intrinsic phase changes $\varepsilon_{p_{r}}(n)$;

- the angle resulting from the phasor rotation at normalized frequency $\frac{2 \pi}{M}(1+\delta)$, with $\delta$ being the off-nominal fractional frequency deviation;

- $\boldsymbol{\eta}_{p}(n)$ is a zero-mean random variable modeling the phase noise due to both sampling jitter and time synchronization uncertainty;

- $\varepsilon_{h_{r}}(n)$ includes the steady-state disturbances due to narrowband (i.e. harmonic and inter-harmonic) components;

- $\boldsymbol{\eta}_{a}(n)$ is a zero-mean additive wideband noise due to the signal acquisition stage of the PMU, although it may also include other possible wideband disturbances superimposed to the input waveform.

Note that the signal model (1) relies on the definition of synchrophasor, as it is reported in the IEEE Standard C37.118.12011 [14]. If, for instance $N$ is odd, the quantities of interest are: the waveform amplitude $A_{r}(0)$, phase $\theta_{r}(0)$, frequency $f_{r}(0)=\frac{1}{2 \pi} \frac{d \theta_{r}}{d t}$ and $R O C O F_{r}(0)=\frac{d f_{r}}{d t}$ at the reference time $t_{r}$. In the rest of this paper, such quantities will be shortly denoted as: $A_{r}, \theta_{r}, f_{r}$ and $R O C O F_{r}$. Since PMUs are also supposed to track the evolution over time of such quantities, $A_{r}(n)$ and $\theta_{r}(n)$ in (1) can be regarded as measurands, while $\boldsymbol{\eta}_{p}(n), \varepsilon_{h_{r}}(n), \boldsymbol{\eta}_{a}(n)$ represent different types of disturbances. Till now, $\boldsymbol{\eta}_{p}(n)$ and $\boldsymbol{\eta}_{a}(n)$ have seldom been included in synchrophasor measurement models because, as stated in the Introduction, the effect of such contributions has been usually assumed to be negligible with respect to both intrinsic amplitude and phase fluctuations or steady-state disturbances. However, if the estimation algorithms are able to mitigate the impact of such contributions, then the effect of noise can become significant. In addition, under the realistic assumption that $\left|\boldsymbol{\eta}_{p}(n)\right|$ is small, amplitude and phase noises tend to sum up. This can be easily shown by expanding (1) as follows:

$$
x_{r}(n) \cong A_{r}(n) \cos \theta_{r}(n)+\varepsilon_{h_{r}}(n)+\boldsymbol{\eta}(n),
$$

where $\boldsymbol{\eta}(n)=\boldsymbol{\eta}_{a}(n)-\boldsymbol{\eta}_{p}(n) A_{r}(n) \sin \theta_{r}(n)$ is the total additive noise given by the sum of two zero-mean and uncorrelated random sequences. Consider that both $\boldsymbol{\eta}_{p}(n)$ and $\boldsymbol{\eta}_{a}(n)$ result from the superimposition of a variety of wideband contributions. Thus, both terms can be reasonably assumed to be normally distributed because of the central limit theorem. As a result, to a first approximation $\boldsymbol{\eta}(n)$ exhibits a Gaussian distribution, as well. It is important to emphasize that $\boldsymbol{\eta}(n)$ includes not only the quantization noise, but also the electrical noise due to the analog front-end, the intrinsic noise superimposed to the input signal and also the phase noise. In particular, it has been recently observed that the phase noise can be so critical as the amplitude noise [25].

\section{B. Noise effect analysis}

The purpose of the theoretical analysis described in this section is to provide proper closed-form expressions describing how synchrophasor, amplitude, phase, frequency and ROCOF estimation uncertainties depend on various quantities including the total Signal-to-Noise Ratio (SNR). This is the ratio between the power of the collected sinewave and the power of the additive noise sequence $\boldsymbol{\eta}(\cdot)$ defined in (2).

In general, the variance of any unbiased estimator of the parameters of a noisy waveform is larger than a threshold. One of the most widely used thresholds is the so-called Cramer-Rao Lower Bound (CRLB) [27]. In a multi-parametric estimation problem, the CRLB expressions actually depend on the number of parameters to be estimated. In the specific context of PMUs, the estimation algorithms have to be natively able to track power waveform variations. To this purpose, four parameters have to be estimated, i.e. synchrophasor amplitude, phase, frequency and ROCOF. Currently, two main categories of estimators exist, i.e. those based on the assumption that the synchrophasor parameters do not change significantly within an observation interval (static models), and those relying on the assumption that all synchrophasor parameters are functions of time (dynamic models). The main difference between them is that the former category does not allow to estimate the ROCOF using a single data record, as the waveform frequency is implicitly assumed to be constant within an observation interval. In such conditions, the CRLBs associated with the estimation of amplitude, phase and frequency only could be derived [28]. However, such CRLBs are not very useful in practice, since they refer to ideal conditions in which the ROCOF is assumed to be zero, which is not very realistic in power systems. For this reason, the CRLBs of unbiased estimators of all four parameters will be considered in the following as a reference for accuracy evaluation and comparison.

In this respect, if $\boldsymbol{\eta}(\cdot)$ is a zero-mean normally distributed white noise over the band $\left[-\frac{f_{s}}{2}, \frac{f_{s}}{2}\right]$, it is shown in Appendix A that the CRLBs for any unbiased estimators of synchrophasor amplitude, phase, frequency and ROCOF are respectively

$$
\begin{gathered}
C R_{A} \cong \frac{A^{2}}{S N R \cdot N} \cong \frac{A^{2}}{2 \cdot S N R_{B}} \frac{1}{C}, \\
C R_{\theta} \cong \frac{9}{4} \frac{1}{S N R \cdot N} \cong \frac{9}{8} \frac{1}{S N R_{B}} \frac{1}{C}, \\
C R_{f} \cong \frac{3}{\pi^{2}} \frac{f_{s}^{2}}{S N R \cdot N^{3}} \cong \frac{3 f_{0}^{2}}{2 \pi^{2}} \frac{1}{S N R_{B}} \frac{1}{C^{3}},
\end{gathered}
$$




$$
C R_{R} \cong \frac{180}{\pi^{2}} \frac{f_{s}^{4}}{S N R \cdot N^{5}} \cong \frac{90 f_{0}^{4}}{\pi^{2}} \frac{1}{S N R_{B}} \frac{1}{C^{5}},
$$

where the three classic PMU design variables, i.e. $f_{s}, N$ and $S N R$ are replaced by $C \cong N / M$ and $S N R_{B}=S N R \cdot \frac{M}{2}$. This choice is justified by the fact that, while $C$ and $S N R_{B}$ are independent, $f_{s}$ and $N$ are not, as $N \cong C \frac{f_{s}}{f_{0}}$ is related to both $C$ and $f_{s}$. Note that, while $C$ affects also estimation latency and responsiveness, $S N R_{B}$ represents the Signal-to-Noise Ratio within $\left[-f_{0}, f_{0}\right]$. Indeed, if $\boldsymbol{\eta}(\cdot)$ is assumed to be white over $\left[-\frac{f_{s}}{2}, \frac{f_{s}}{2}\right]$, the amount of noise power included within $\left[-f_{0}, f_{0}\right]$ is a fraction $\frac{2 f_{0}}{f_{s}}=\frac{2}{M}$ of the total wideband noise. Hence, $\left[-f_{0}, f_{0}\right]$ can be also regarded as the minimum possible band of an ideal anti-aliasing filter, thus justifying the physical meaning of $S N R_{B}$. Equations (3)-(6) show compactly that the effect of noise can be kept under control by increasing $C$ or the Signal-to-Noise Ratio within the band $\left[-f_{0}, f_{0}\right]$.

It is important to remind that when just amplitude, phase and frequency can be estimated using a single data record (i.e. in the case of estimation algorithms based on synchrophasor static models), the corresponding CRLBs are partially different from (3)-(5). In particular, while the CRLBs for amplitude and frequency estimation are the same as (3) and (5), respectively, the CRLB associated with phase estimation of a real-valued sinusoid in the center of the considered observation interval is $\frac{4}{9}$ of (4) [28].

Unfortunately, expressions (3)-(6) are not immediately suitable for a comparison with the requirements of the IEEE Standards. In fact, different accuracy parameters for synchrophasor, frequency and ROCOF measurements are defined in [14], i.e.

- the Total Vector Error (TVE)

$$
T V E_{r}=\frac{\left|\hat{A}_{r} e^{j \hat{\theta}_{r}}-A_{r} e^{j \theta_{r}}\right|}{\left|A_{r} e^{j \theta_{r}}\right|}
$$

- the Frequency Error (FE)

$$
F E_{r}=\left|\hat{f}_{r}-f_{r}\right|
$$

- $\quad$ and the Rate of Frequency Error (RFE)

$$
R F E_{r}=\left|R \widehat{O C O} F_{r}-R O C O F_{r}\right| .
$$

where index $r$ refers the $r$-th observation interval and the "hat" symbol denotes the estimated quantities. Observe that the values of $T V E_{r}, F E_{r}$ and $R F E_{r}$ in the presence of wideband noise change randomly from record to record. Therefore, (7)(9) can be regarded as random variables. Since they are nonnegative by definition, their mean value is larger than zero, even when the estimators of amplitude, phase, frequency or ROCOF are statistically unbiased. Thus, proper relationships between (3)-(6) and the mean squared values of (7)-(9), namely the Mean Square Errors (MSEs) of $T V E_{r}, F E_{r}$ and $R F E_{r}$, have to be determined to quantify the best achievable estimation accuracy. As far as $T V E_{r}$ is concerned, it ensues from (3)-(4) (see Appendix B) that a lower bound to its mean squared value is approximately

$$
L B_{T V E} \cong 2 \frac{C R_{A}}{A^{2}}+\frac{5}{9} C R_{\theta} \cong \frac{13}{8} \frac{1}{S N R_{B}} \cdot \frac{1}{C} .
$$

The derivation of the mean square values of $F E_{r}$ and $R F E_{r}$ is instead straightforward, as it relies on the basic properties of such random variables. In fact, since both frequency and ROCOF estimates can be assumed to be almost normally distributed, then the probability density functions of $F E_{r}$ and $R F E_{r}$ are almost half-normal and their mean square values coincide with the variance of frequency and ROCOF estimates, respectively. Thus, the corresponding MSE lower bounds are simply

$$
L B_{F E}=C R_{f} \text { and } L B_{R F E}=C R_{R} .
$$

Observe that $L B_{T V E}, L B_{F E}$ and $L B_{R F E}$ are squared quantities. So, their respective square root values should be used in order to enable a dimensionally consistent comparison with the P Class or M Class limits reported in the IEEE Standards. In particular, the upper endpoints of the confidence intervals associated with $T V E_{r}, F E_{r}$ and $R F E_{r}$ (in the following shortly referred to as maximum uncertainties for brevity) are certainly proportional to $\sqrt{L B_{T V E}}, \sqrt{L B_{F E}}$ and $\sqrt{L B_{R F E}}$, and can be defined as

$$
\begin{aligned}
U_{T V E} & =\beta_{T V E} \cdot \sqrt{L B_{T V E}}, \\
U_{F E} & =\beta_{F E} \cdot \sqrt{L B_{F E}}, \\
U_{R F E} & =\beta_{R F E} \cdot \sqrt{L B_{R F E}},
\end{aligned}
$$

where coefficients $\beta_{T V E}>0, \beta_{F E}>0$ and $\beta_{R F E}>0$ depend on the Equivalent Noise Bandwidth (ENBW) of the adopted window function, the features of the estimation algorithm (as it will be shown in Section III) and, of course, the level of confidence chosen to define the maximum uncertainty (e.g. $95 \%, 98 \%$ or $99.7 \%$ ). It is important to highlight that, once the confidence level is given, the lowest possible $\beta$ coefficients correspond to those of unbiased estimators of synchrophasor amplitude, phase, frequency and ROCOF that reach the respective CRLBs. No other estimation algorithms can ensure lower values for these coefficients when all four quantities above have to be estimated using the same data record. For instance, if a $99.7 \%$ level of confidence is considered, it follows that $\beta_{T V E} \geq 2.6$ (this is slightly larger than the $99.7 \%$ percentile of a Rayleigh distribution for the reasons explained in Appendix B), while $\beta_{F E} \geq 3$ and $\beta_{R F E} \geq 3$, as they coincide with the 99.7\% percentile of half-normal distributions.

\section{SimUlation RESUlTS}

The results reported in this Section confirm the previous theoretical analysis and show the impact of wideband noise in different testing conditions specified in the IEEE Standards. In the following, the theoretical maximum uncertainty values provided by (12) are compared with those obtained via Monte Carlo simulations using three state-of-the-art techniques for synchrophasor, frequency and ROCOF estimation, i.e. the Generalized Taylor Weighted Least Squares (GTWLS) algorithm [10], the Interpolated Dynamic Discrete Fourier Transform $\left(\mathrm{IpD}^{2} \mathrm{FT}\right)$ technique [17] and the Interpolated Discrete Fourier Transform (IpDFT) estimator [4], [5]. A common feature of all algorithms is their ability to estimate and to 


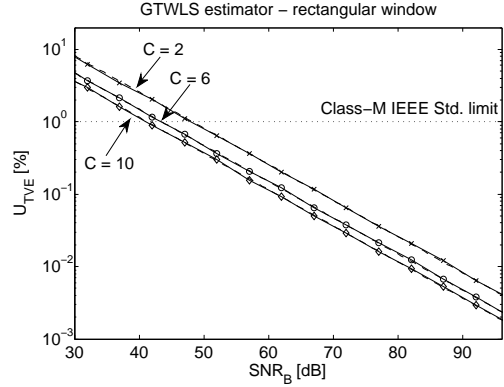

(a)

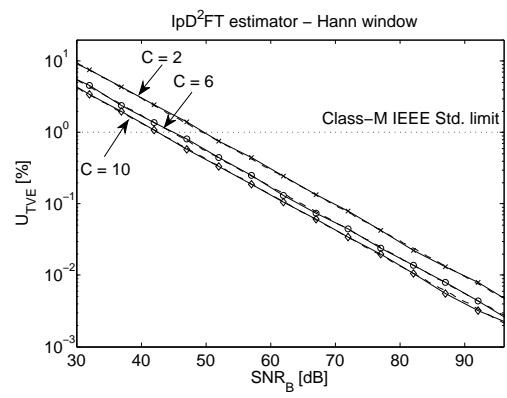

(d)

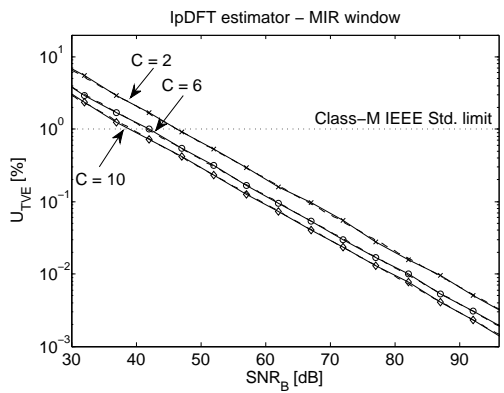

(g)

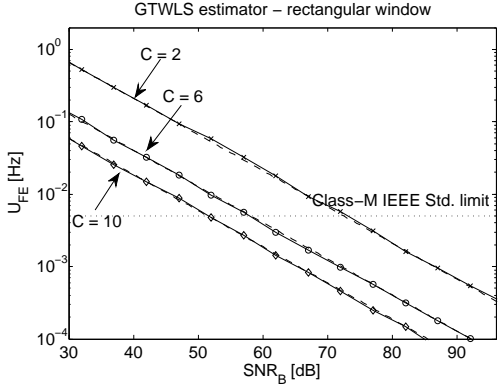

(b)

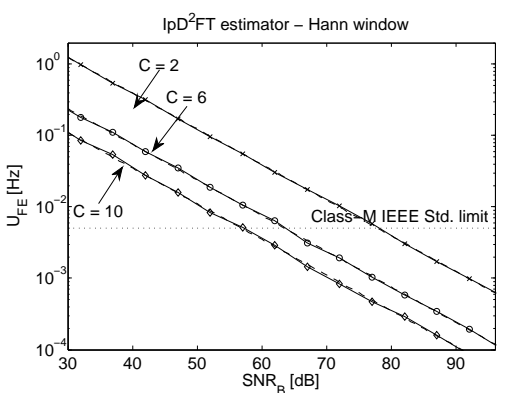

(e)

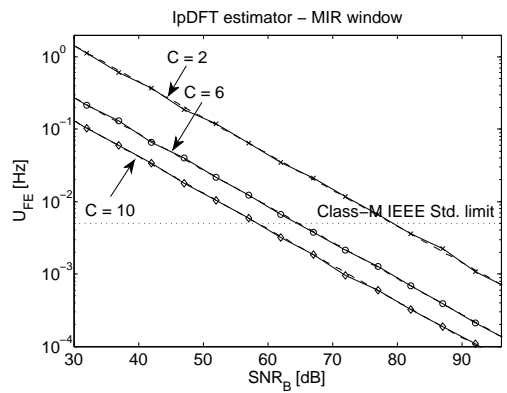

(h)

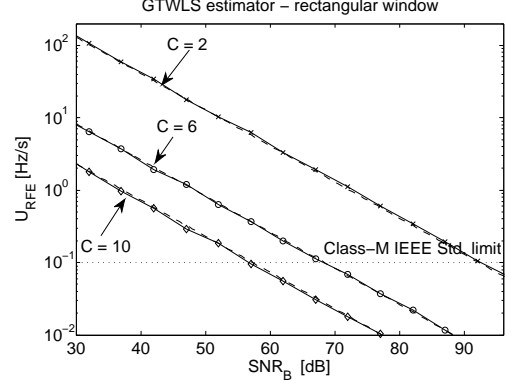

(c)

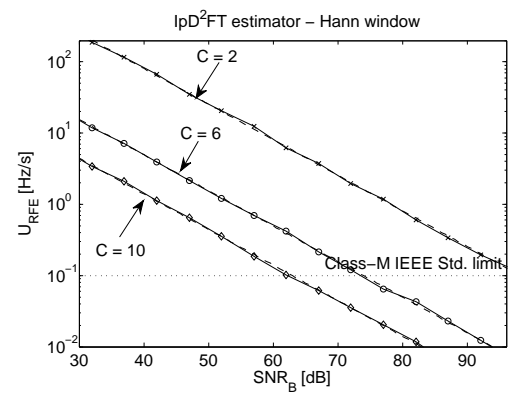

(f)

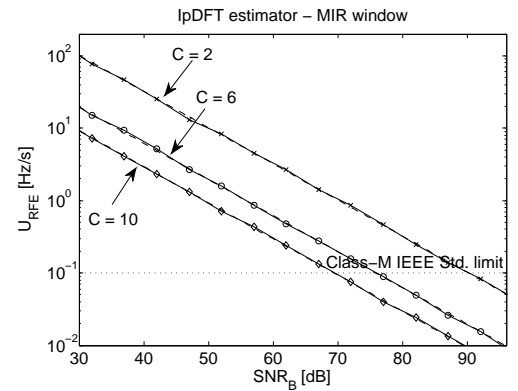

(i)

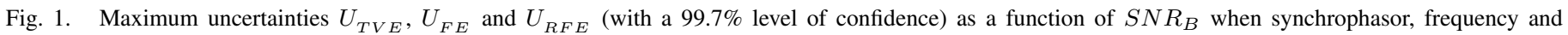

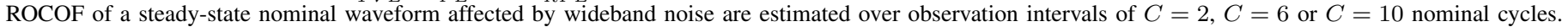

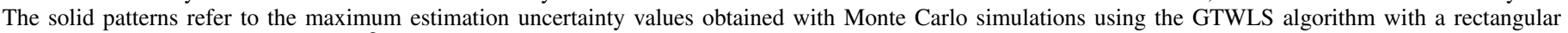

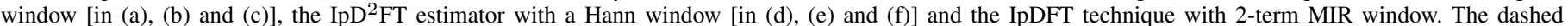

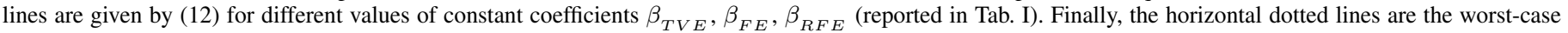
Class M limits reported in the IEEE Standards in steady-state conditions.

compensate for possible estimation errors due to static deviations of the fundamental frequency. However, only the first two estimators rely on a dynamic synchrophasor model. This is obtained by truncating to the second order the Taylor's series expansion with respect to time of the synchrophasor function. The IpDFT instead is based on a static synchrophasor model. In fact, the signal fundamental frequency is estimated by interpolating the samples within the mainlobe of the windowed DFT of the collected waveform [29]. The ROCOF cannot be estimated directly from model parameters, but it can be obtained from the first-order finite difference of the frequency values estimated over two subsequent observation intervals (e.g. shifted by 1 waveform cycle). This approach requires using one additional cycle, i.e. further information with respect to that assumed to derive (3)-(6). Despite this, the IpDFT algorithm has been considered in the simulation analysis for the sake of completeness.

Fig. 1 shows, on a logarithmic scale, the trends of the maximum uncertainties $U_{T V E}, U_{F E}$ and $U_{R F E}$ (with a $99.7 \%$ level of confidence) when a $50-\mathrm{Hz}$ sine-wave with amplitude $A=1$ p.u. sampled at $f_{s}=3.2 \mathrm{kHz}$ (i.e. $M=64$ ) is affected by different levels of wideband noise. All curves are plotted as a function of $S N R_{B}$ (expressed in $\mathrm{dB}$ ) for $C=2, C=6$ and $C=10$ nominal cycles and using three different algorithms, i.e. the GTWLS algorithm with a rectangular window in Fig. 1(a)-(c), the $\mathrm{IpD}^{2} \mathrm{FT}$ technique with the Hann window in Fig. 1(d)-(f) and the IpDFT estimator with the 2-term Maximum Image Rejection (MIR) window 
TABLE I. MEAN VALUES OF THE $\beta$ COEFFICIENTS ASSOCIATED WITH THE GTWLS, IPD ${ }^{2}$ FT AND IPDFT ALGORITHMS USING DIFFERENT WINDOW FUNCTIONS OVER OBSERVATION INTERVALS OF 2,6 , AND 10 NOMINAL CYCLES. ALL VALUES HAVE BEEN ESTIMATED IN NOMINAL, STEADY-STATE CONDITIONS

\begin{tabular}{|c|c|c|c|c|c|c|c|c|c|c|c|c|c|}
\hline \multirow{2}{*}{ Algorithm } & \multirow{2}{*}{ No. cycles } & \multicolumn{3}{|c|}{ Rectangular window } & \multicolumn{3}{|c|}{ Hann window } & \multicolumn{3}{|c|}{ 2-term MIR window } & \multicolumn{3}{|c|}{ 3-term MSD window } \\
\hline & & $\hat{\beta}_{T V E}$ & $\hat{\beta}_{F E}$ & $\hat{\beta}_{R F E}$ & $\hat{\beta}_{T V E}$ & $\hat{\beta}_{F E}$ & $\hat{\beta}_{R F E}$ & $\hat{\beta}_{T V E}$ & $\hat{\beta}_{F E}$ & $\hat{\beta}_{R F E}$ & $\hat{\beta}_{T V E}$ & $\hat{\beta}_{F E}$ & $\hat{\beta}_{R F E}$ \\
\hline \multirow{3}{*}{ GTWLS } & $C=2$ & 2.8 & 3.1 & 3.2 & 3.6 & 6.4 & 8.0 & 3.5 & 6.0 & 7.2 & 4.0 & 9.0 & 16.0 \\
\hline & $C=6$ & 2.9 & 3.0 & 3.0 & 3.5 & 6.5 & 8.2 & 3.5 & 6.2 & 8.4 & 4.1 & 9.4 & 16.0 \\
\hline & $C=10$ & 2.9 & 3.0 & 3.0 & 3.6 & 6.4 & 8.4 & 3.6 & 6.3 & 8.3 & 4.1 & 9.4 & 16.0 \\
\hline \multirow{3}{*}{$\mathrm{IpD}^{2} \mathrm{FT}$} & $C=2$ & 3.0 & 3.9 & 3.3 & 3.4 & 5.7 & 6.1 & 3.2 & 5.5 & 6.0 & 3.8 & 7.4 & 10.2 \\
\hline & $C=6$ & 2.9 & 3.8 & 3.1 & 3.4 & 5.7 & 5.8 & 3.2 & 5.7 & 5.7 & 3.6 & 7.3 & 9.6 \\
\hline & $C=10$ & 2.9 & 3.8 & 3.1 & 3.4 & 5.8 & 5.8 & 3.3 & 5.6 & 5.7 & 3.6 & 7.3 & 9.4 \\
\hline \multirow{3}{*}{ IpDFT } & $C=2$ & 2.0 & 5.5 & 2.2 & 2.5 & 6.5 & 2.4 & 2.3 & 6.5 & 2.4 & 2.6 & 8.3 & 3.0 \\
\hline & $C=6$ & 2.0 & 5.1 & 6.1 & 2.5 & 6.7 & 7.2 & 2.3 & 6.6 & 7.3 & 2.6 & 8.2 & 8.5 \\
\hline & $C=10$ & 2.0 & 5.0 & 10.0 & 2.5 & 6.8 & 12.3 & 2.3 & 6.7 & 12.3 & 2.6 & 8.3 & 15.2 \\
\hline
\end{tabular}

TABLE II. MEAN VALUES AND RANGES OF VARIATION OF THE $\beta$ COEFFICIENTS ASSOCIATED WITH DIFFERENT ALGORITHMS AND WINDOW FUNCTIONS ESTIMATED IN FOUR TESTING CONDITIONS SPECIFIED IN THE IEEE STANDARDS C37.118.1-2011 AND C37.118.1A-2014 OVER OBSERVATION INTERVALS OF 2,6 , AND 10 NOMINAL CYCLES.

\begin{tabular}{|c|c|c|c|c|c|c|c|c|c|c|c|c|}
\hline \multirow[b]{2}{*}{ Algorithm } & \multicolumn{3}{|c|}{ Rectangular window } & \multicolumn{3}{|c|}{ Hann window } & \multicolumn{3}{|c|}{ 2-term MIR window } & \multicolumn{3}{|c|}{ 3-term MSD window } \\
\hline & $\hat{\beta}_{T V E}$ & $\hat{\beta}_{F E}$ & $\hat{\beta}_{R F E}$ & $\hat{\beta}_{T V E}$ & $\hat{\beta}_{F E}$ & $\hat{\beta}_{R F E}$ & $\hat{\beta}_{T V E}$ & $\hat{\beta}_{F E}$ & $\hat{\beta}_{R F E}$ & $\hat{\beta}_{T V E}$ & $\hat{\beta}_{F E}$ & $\hat{\beta}_{R F E}$ \\
\hline GTWLS & $2.8 \pm 0.2$ & $3.0 \pm 0.2$ & $3.0 \pm 0.3$ & $3.5 \pm 0.2$ & $6.2 \pm 0.3$ & $8.1 \pm 0.5$ & $3.5 \pm 0.1$ & $6.2 \pm 0.5$ & $7.9 \pm 0.6$ & $4.1 \pm 0.3$ & $9.2 \pm 0.9$ & $16 \pm 2$ \\
\hline $\mathrm{IpD}^{2} \mathrm{FT}$ & $2.9 \pm 0.7$ & $3.8 \pm 0.5$ & $3.0 \pm 2$ & $3.4 \pm 0.6$ & $5.7 \pm 0.4$ & $6 \pm 2$ & $3.3 \pm 0.7$ & $5.6 \pm 0.5$ & $6.0 \pm 2$ & $3.8 \pm 0.3$ & $7.3 \pm 0.6$ & $10 \pm 3$ \\
\hline
\end{tabular}

in Fig. 1(g)-(i) [30]. The solid lines refer to the results of Monte Carlo simulations over 2000 runs in which the initial phase and the additive wideband noise change randomly. The dashed lines represent the respective theoretical values given by (12) for different sets of constant coefficients $\beta_{T V E}, \beta_{F E}$ and $\beta_{R F E}$ (further details on these values are explained in the last part of this Section). Finally, the horizontal dotted lines represent the Class $\mathrm{M}$ limits reported in the IEEE Amendment C37.118.1a-2014 in steady-state conditions [16]. Observe that all theoretical lines are almost perfectly overlapped to the simulated patterns for both short and long observation intervals. These results confirm the correctness of (12). Note also that by increasing the value of $C$, estimation accuracy improves in all cases, as expected.

The results in Fig. 1(a)-(c) obtained using the GTWLS algorithm with a rectangular window are particularly interesting, as this approach relies on classic least squares minimization, which provides maximum likelihood estimation [28]. Nevertheless, this window is hardly used in practice because of its relevant scalloping loss and the significant magnitude of its spectral side-lobes [30].

It is important to highlight that the values of $U_{T V E}, U_{F E}$ and $U_{R F E}$ can be larger and, sometimes, even much larger than the limits reported in the Standard even for quite large values of $S N R_{B}$. This is true especially for frequency and, above all, for ROCOF estimation. For example, in Class M steady-state conditions, for $C=2$ and $S N R_{B}=67 \mathrm{~dB}$ (this value can be achieved, for instance, with $M=64$ and $S N R=52 \mathrm{~dB}$ ) the effect of noise on TVE is clearly one order of magnitude smaller than the $1 \%$ Standard limit (i.e. negligible). However, for the same values of $C$ and $S N R_{B}$, both the FE and the RFE Standard limits are violated (the latter by about one order of magnitude). This situation suggests that, if the effective resolution of the data acquisition stage cannot be improved any further, either a greater value of $M$ (and consequently a higher sampling rate) is used (provided that the noise is white over the band $\left[-\frac{f_{s}}{2}, \frac{f_{s}}{2}\right]$ ), or a much larger number of cycles has to be observed. However, both these solutions may pose problems in terms of processing latency and algorithm responsiveness.

Coefficients $\beta_{T V E}, \beta_{F E}$ and $\beta_{R F E}$ play a key role to analyze and to compare the noise sensitivity of different algorithms. In general, the values of such coefficients can be hardly determined analytically. However, in the case of nominal, steady-state conditions, they can be easily found numerically. To this purpose, first the values of $\hat{U}_{T V E}, \hat{U}_{F E}$ and $\hat{U}_{R F E}$ for a given algorithm and level of confidence (e.g. 99.7\%) are estimated through Monte Carlo simulations by changing randomly both initial phase and wideband noise power over a reasonably large number of records. Then, the values of $\beta$ coefficients can be estimated as follows, i.e.

$$
\begin{gathered}
\beta_{T V E} \cong \hat{\beta}_{T V E}=\frac{1}{L} \sum_{l=1}^{L} \frac{\hat{U}_{T V E}(l)}{\sqrt{L B_{T V E}}} \\
\beta_{F E} \cong \hat{\beta}_{F E}=\frac{1}{L} \sum_{l=1}^{L} \frac{\hat{U}_{F E}(l)}{\sqrt{L B_{F E}}} \\
\beta_{R F E} \cong \hat{\beta}_{R F E}=\frac{1}{L} \sum_{l=1}^{L} \frac{\hat{U}_{R F E}(l)}{\sqrt{L B_{R F E}}} .
\end{gathered}
$$

where the averages on the rightmost side of (13) are performed over $L$ different levels of $S N R_{B}$ in order to smooth possible numerical fluctuations. 
Tab. I reports the values of the $\beta$ coefficients associated with the GTWLS, IpD ${ }^{2}$ FT and IpDFT algorithms for four different types of windows (i.e. rectangular, Hann, 2-term MIR, and 3-term Maximum Side-lobe Decay - MSD) estimated over observation intervals of 2, 6, and 10 nominal waveform cycles, respectively, with records of 2000 runs each, and for $L=20$ values of $S N R_{B}$ in the range $[30,100] \mathrm{dB}$. Some of these coefficients have been also used in Fig. 1(a)-(i) to verify (12). The results in Tab. I are interesting for a variety of reasons. First, they show that all $\beta$ coefficients tend to grow as the number of terms of the window functions increases, since their ENBWs also grow accordingly. However, for a given number of terms, the impact of the window shape is minor, as its ENBW does not change significantly. For instance, the values obtained with the MIR and Hann windows are quite close to each other. Note also that, in most cases, for a given algorithm and for a fixed value of $C$, the values of $\beta_{R F E}$ are usually larger than those of $\beta_{F E}$ and much larger than those of $\beta_{T V E}$. This confirms that the statistical efficiency of ROCOF estimators is generally poorer than in the case of syncrophasor amplitude, phase and frequency estimation. Moreover, the values of $\beta_{R F E}$ increase faster than $\beta_{T V E}$ and $\beta_{F E}$ as a function of the number of window terms. This trend is particularly clear when the GTWLS algorithm is used. Probably this is due to the fact that this approach fully exploits all the noisy data collected in each observation interval. IpD ${ }^{2} \mathrm{FT}$ and IpDFT instead rely just on the samples within the spectrum main lobe of the chosen window. Therefore, noise sensitivity is expected to be lower since those samples contain most of the signal power and just a limited fraction of noise power.

It is worth noticing that the values of $\beta_{T V E}$ in the case of the IpDFT are sometimes smaller than the minimum expected value (i.e. about 2.6). This result is correct since the IpDFT is conceived to estimate only three waveform parameters (i.e. amplitude, phase and frequency). Therefore, when the IpDFT with a rectangular window is used, amplitude and phase estimation variances approach the respective CRLBs for a three-parameter estimation problem [28]. Given that, as explained in Section II-B, the CRLB for phase estimation is $\frac{4}{9}$ of (4), the lower bound to the root mean square value associated with $T V E_{r}$ is potentially smaller than (10), i.e. $2 \frac{C R_{A}}{A^{2}}=\frac{1}{S N R_{B}} \cdot \frac{1}{C}$. However, in this case ROCOF estimation is ignored completely, whereas the beta coefficients resulting from (13) are obtained under the assumption that also the ROCOF has to be considered. Consequently, the values of $L B_{T V E}, L B_{F E}$ and $L B_{R F E}$ related to the four-parameter estimation problem are used at the denominators of (13). This explains why, when the IpDFT is used, the values of $\beta_{T V E}$ are generally smaller than those obtained with the estimators based on the dynamic model. Moreover, in the IpDFT case the values of $\beta_{R F E}$ strongly depend on the observation interval length, since the finite difference approach used to estimate the ROCOF relies on two data records of variable size shifted by one waveform cycle.

A further point that deserves attention concerns with the effect of other disturbances on top of wideband noise. Even if expressions (10)-(11) hold only when sine-waves with a constant ROCOF are corrupted by additive wideband noise, in realistic operating scenarios further disturbances may affect a power waveform. Therefore, a proper robustness analysis is needed to evaluate to what extent the combination of noise and such additional disturbances may affect parameter estimation uncertainty.

Even if the superimposition principle does not hold rigorously, the wideband noise magnitude, for typical values of $S N R_{B}$, is small enough to assume that all estimators can be linearized around the estimated values. This approach is very similar to the one adopted in [31] to evaluate the propagation of uncertainty when nonlinear measurement models are used. However, since the influence of such disturbances can be only partially compensated before applying (13), in realistic conditions the $\beta$ coefficients may exhibit significant fluctuations around the respective expected values. In the following, the ranges of variation of the $\beta$ coefficients for the three algorithms considered have been computed in four testing conditions specified in the IEEE Standards, i.e.

- the Class-M frequency ramp test, i.e. with the fundamental frequency changing linearly from $45 \mathrm{~Hz}$ to 55 $\mathrm{Hz}$ at $1 \mathrm{~Hz} / \mathrm{s}$;

- steady-state tests with the waveform fundamental frequency affected by static deviations within $\pm 10 \%$ of the nominal value;

- amplitude modulation (AM) tests, with the fundamental waveform subjected to sinusoidal oscillations of frequency up to $5 \mathrm{~Hz}$ and amplitude within $\pm 10 \%$ of the nominal value;

- and, finally, phase modulation (PM) tests, with the fundamental waveform subjected to sinusoidal oscillations of frequency up to $5 \mathrm{~Hz}$ and amplitude within $\pm 0.1 \mathrm{rad}$. Tab. II summarizes the mean values as well as the ranges of variation of $\hat{\beta}_{T V E}, \hat{\beta}_{F E}$ and $\hat{\beta}_{R F E}$ for the same algorithms and windows shown in Tab. I over all the testing conditions listed above. Of course, unlike Tab. I, the ranges of variation are no longer negligible, as they include both the residual effect of disturbance-specific uncertainty contributions and the dependence on observation interval length. Despite this, in most cases, the average values almost coincide with the those reported in Tab. I, as expected. Also, the maximum relative uncertainties of the $\beta$ parameters are typically $\pm 10 \%$ for TVE and FE and up to about $\pm 30 \%$ for RFE (except in the IpDFT case for the reasons explained above).

\section{DESIGN GUIDELINES OF THE DATA ACQUISITION STAGE OF A PMU}

The results of the theoretical analysis reported in Section II-B can be used to keep under control the impact of wideband noise on synchrophasor amplitude, phase, frequency and ROCOF estimation. From a design perspective, this can be done by properly setting three key parameters of the acquisition stage of a PMU, i.e. the observation interval length $C$ (which affects both algorithm responsiveness and estimation accuracy), the sampling rate $f_{s}$ and the PMU effective resolution expressed in $E N O B$. In particular, $C, f_{s}$ and $E N O B$ must be large enough to ensure that, for a given algorithm,

$$
U_{T V E} \leq \frac{\tau_{T V E_{i}}}{F}, \quad U_{F E} \leq \frac{\tau_{F E_{i}}}{F}, \quad U_{R F E} \leq \frac{\tau_{R F E_{i}}}{F}
$$


where $\tau_{T V E}, \tau_{F E_{i}}$ and $\tau_{R F E}$ for $i \in\{P, M\}$ are the most stringent $\mathrm{P}$ Class or M Class limits among those specified in the IEEE Standards for different testing conditions [14], [16]. Moreover, $F \geq 1$ is an arbitrary constant factor to be set large enough to keep the noise-related uncertainty well below the related limits. However, increasing $F$ too much could lead to excessive (i.e. infeasible) requirements in terms of sampling rate and/or effective resolution. As a rule of thumb, $F=3$ provides a reasonable trade-off between noise impact reduction and PMU design feasibility.

While at a glance, the three conditions (14) must be satisfied independently, in practice it is shown in Appendix $\mathrm{C}$ that for reasonably small values of $C$, i.e. when

$$
1 \leq C \leq C_{i}^{*} \cong\left\lfloor 2 \sqrt{15} f_{0} \frac{\beta_{R F E}}{\beta_{F E}} \frac{\tau_{F E_{i}}}{\tau_{R F E_{i}}}\right\rfloor \quad i \in\{P, M\},
$$

(where $\lfloor\cdot\rfloor$ denotes the floor operator) the constraint on RFE prevails on the others. In the current edition of the IEEE Standards, $\tau_{F E_{P}}=\tau_{F E_{M}}=5 \mathrm{mHz}, \tau_{R F E_{P}}=0.4 \mathrm{~Hz} / \mathrm{s}$ and $\tau_{R F E_{M}}=0.1 \mathrm{~Hz} / \mathrm{s}$. Therefore, given that usually $\beta_{R F E} \geq \beta_{F E}$ (see Tabs. I-II), it follows from (15) that $C_{P}^{*} \geq 4$ and $C_{M}^{*} \geq 19$ for $f_{0}=50 \mathrm{~Hz}$, while $C_{P}^{*} \geq 5$ and $C_{M}^{*} \geq 23$ for $f_{0}=60 \mathrm{~Hz}$. These values are safely larger than the typical observation interval lengths of $\mathrm{P}$ Class and M Class PMUs, respectively. As a consequence, choosing the PMU data acquisition parameters just on the basis of the impact of wideband noise on ROCOF estimation is a sensible approach to simplify the design procedure. To this end, two basic steps are needed.

1) First, once the synchrophasor estimation algorithm to be implemented is chosen, coefficients $\hat{\beta}_{T V E}, \hat{\beta}_{F E}$ and $\hat{\beta}_{R F E}$ have to be computed in nominal steady-state conditions, as explained in Section III.

2) Then, for a given observation interval length $C \leq C_{i}^{*}$, suitable pairs of $f_{s}$ and $E N O B$ values have to be arbitrarily chosen among those satisfying condition (see Appendix C)

$$
f_{s} \geq \frac{\hat{\beta}_{R F E}^{2}}{10^{\frac{6.02 \cdot E N O B+1.76}{10}}} \frac{2 f_{0} \cdot \Gamma_{i}}{C^{5}}
$$

where $\Gamma_{i}=\frac{90 f_{0}^{4}}{\pi^{2}} \frac{F^{2}}{\tau_{R F E_{i}}^{2}}$, for $i \in\{P, M\}$, is a constant value.

Tabs. III(a)-(b) shows some examples of $f_{s}$ lower bounds (expressed in $\mathrm{kHz}$ ) given by (16) for some typical values of $\hat{\beta}_{R F E}, C$ and $E N O B$ with $F=3, f_{0}=50 \mathrm{~Hz}$, and for P Class and M Class PMUs, respectively. Observe that the values of $C$ and $E N O B$ chosen to build Tabs. III(a)-(b) are purposely different, since P Class PMUs are supposed to be faster and less accurate than M Class PMUs. In general, it is advisable to choose a balanced configuration in terms of $f_{s}$ and $E N O B$ for a given value of $C$. Indeed, as a rule of thumb, increasing the sampling rate usually degrades the effective resolution of a data acquisition system and vice versa. Moreover, larger values of $f_{s}$ and $E N O B$ usually imply higher hardware design costs. If the observation interval length is adjustable, but the sampling rate is not, the values $f_{s}$ and $E N O B$ should be chosen on
TABLE III. MINIMUM $f_{s}$ VALUES (IN KHZ) FOR WHICH THE SYNCHROPHASOR PARAMETERS ESTIMATION UNCERTAINTIES DUE TO WIDEBAND NOISE ARE SMALLER THAN AT MOST 1/3 OF THE STRICTEST P CLASS (A) OR M CLASS (B) LIMITS REPORTED IN THE IEEE STANDARDS. THE SAMPLING RATES RESULT FROM (16) FOR SOME TYPICAL VALUES OF

\begin{tabular}{|c|c|c|c|c|c|c|c|c|c|}
\hline \multirow{3}{*}{$\hat{\boldsymbol{\beta}}_{R F E}$} & \multicolumn{3}{|c|}{$C=1$} & \multicolumn{3}{|c|}{$C=2$} & \multicolumn{3}{|c|}{$C=3$} \\
\hline & \multicolumn{3}{|c|}{$E N O B$} & \multicolumn{3}{|c|}{$E N O B$} & \multicolumn{3}{|c|}{$E N O B$} \\
\hline & 12 & 13 & 14 & 12 & 13 & 14 & 12 & 13 & 14 \\
\hline $\begin{array}{l}3.0 \\
\end{array}$ & 115 & 29 & 7.2 & 3.6 & 0.9 & 0.2 & 0.5 & 0.1 & 0.1 \\
\hline 4.5 & 258 & 65 & 16 & 8.1 & 2.0 & 0.5 & 1.1 & 0.3 & 0.1 \\
\hline 6.0 & 460 & 115 & 29 & 14 & 3.6 & 0.9 & 1.9 & 0.5 & 0.1 \\
\hline 7.5 & 718 & 180 & 45 & 22 & 5.6 & 1.4 & 3.0 & 0.7 & 0.2 \\
\hline 9.0 & 1034 & 259 & 65 & 32 & 8.1 & 2.0 & 4.3 & 1.1 & 0.3 \\
\hline 10.5 & 1407 & 352 & 88 & 44 & 11 & 2.7 & 5.8 & 1.4 & 0.4 \\
\hline 12 & 1838 & 456 & 115 & 57 & 14 & 3.6 & 7.6 & 1.9 & 0.5 \\
\hline 13.5 & 2326 & 582 & 145 & 73 & 18 & 4.5 & 10 & 2.4 & 0.6 \\
\hline 15 & 2872 & 718 & 180 & 90 & 22 & 5.6 & 12 & 3.0 & 0.7 \\
\hline
\end{tabular}
$\hat{\beta}_{R F E}, C$ AND $E N O B$ WHEN $f_{0}=50 \mathrm{~Hz}$.

\begin{tabular}{|c|c|c|c|c|c|c|c|c|c|}
\hline \multirow{3}{*}{$\hat{\boldsymbol{\beta}}_{R F E}$} & \multicolumn{3}{|c|}{$C=2$} & \multicolumn{3}{|c|}{$C=3$} & \multicolumn{3}{|c|}{$C=4$} \\
\hline & \multicolumn{3}{|c|}{$E N O B$} & \multicolumn{3}{|c|}{$E N O B$} & \multicolumn{3}{|c|}{$E N O B$} \\
\hline & 13 & 14 & 15 & 13 & 14 & 15 & 13 & 14 & 15 \\
\hline 3.0 & 14 & 3.6 & 1.0 & 1.9 & 0.5 & 0.1 & 0.4 & 0.1 & 0.1 \\
\hline 4.5 & 32 & 8.1 & 2.0 & 4.3 & 1.1 & 0.3 & 1.0 & 0.3 & 0.1 \\
\hline 6.0 & 57 & 14 & 3.6 & 7.6 & 1.9 & 0.5 & 1.8 & 0.4 & 0.1 \\
\hline 7.5 & 90 & 22 & 5.6 & 12 & 3.0 & 0.7 & 2.8 & 0.7 & 0.2 \\
\hline 9.0 & 129 & 32 & 8.1 & 17 & 4.3 & 1.1 & 4.0 & 1.0 & 0.3 \\
\hline 10.5 & 176 & 44 & 11 & 23 & 5.8 & 1.4 & 5.5 & 1.4 & 0.3 \\
\hline 12 & 230 & 57 & 14 & 30 & 7.6 & 1.9 & 7.2 & 1.8 & 0.4 \\
\hline 13.5 & 291 & 73 & 18 & 38 & 9.6 & 2.4 & 9.1 & 2.3 & 0.6 \\
\hline 15 & 359 & 90 & 22 & 47 & 12 & 3.0 & 11 & 2.8 & 0.7 \\
\hline
\end{tabular}

(a)

(b)

the basis of the minimum allowed observation interval length. Indeed, with longer observation intervals the impact of noise certainly decreases and, eventually, becomes negligible in any case as confirmed by (3)-(6).

Observe that some cells in Tabs. III(a)-(b) are shadowed, as they correspond to results which are not meaningful in practice for two different reasons. The cells in the bottom left corner of either Table are shadowed as the related sampling rates are higher than $100 \mathrm{kHz}$. Indeed, the PMU processing time grows with the total number of acquired samples $N$, which, in turn, increases with $f_{s}$. Therefore, if $f_{s}>100 \mathrm{kHz}$, real-time data reporting rates up to 50 or 60 frames per second (or more, as required in the IEEE Standards) can become very difficult of even impossible to achieve with existing technologies. Moreover, noise power reduction through oversampling makes sense only if the noise itself is approximately white over the whole band $\left[-\frac{f_{s}}{2}, \frac{f_{s}}{2}\right]$. If instead the noise power spectral density is band-limited (e.g. because of the input anti-aliasing filter), oversampling the input waveform not only increases the processing time, but it does not provide significant accuracy improvements either.

Conversely, the cells in the top right corner of Tabs. III(a)- 
(b) are shadowed because the $f_{s}$ values are excessively low. So, the estimation results, although quite unaffected by wideband noise, could be strongly perturbed by the aliasing of residual harmonic and inter-harmonic disturbances.

In order to clarify with an example how the results of this Section could be useful in practice, let us assume that the $\mathrm{IpD}^{2} \mathrm{FT}$ algorithm based on the Hann window (i.e. with $\hat{\beta}_{R F E}=6$ ) has to be implemented in a P Class PMU. If the shortest observation interval is equal to $C=1$ cycle, then a data acquisition system with a sampling rate of at least $29 \mathrm{kHz}$ and $E N O B=14$ bits is good enough to make the effect of noise is negligible. However, if $C=2$, both these requirements can be further relaxed.

\section{COnClusions}

This paper presents a comprehensive analysis of the effect of wideband noise on synchrophasor, amplitude, phase, frequency and ROCOF estimation accuracy. The proposed analysis shows that the impact of wideband noise may become relevant, and it is particularly critical for frequency and, above all, ROCOF estimation. In particular, data acquisition systems with 1213 effective bits and a sampling rate of some $\mathrm{kHz}$ could be too low to make the effect of noise negligible when very short observation intervals are used. This problem can be solved in different complementary ways, i.e. i) by using longer observation intervals (but this may affect estimation responsiveness and processing time); ii) by increasing the sampling rate (but only if the total noise is white over the whole Nyquist band and only till when the processing time meets real-time requirements) or iii) by increasing the number of effective bits of a PMU. In practice, the wise combination of these three approaches is expected to provide the most sensible solution, which however may depend also on additional contour conditions (e.g. budget or market constraints). The design guidelines described in this paper can help engineers to achieve their specific goals as far as the effect of wideband noise on PMU measurement accuracy is concerned.

\section{APPENDIX A}

DERIVATION OF EXPRESSIONS (3)-(6)

Let us assume that both $\varepsilon_{a_{r}}(\cdot)$ and $\varepsilon_{h_{r}}(\cdot)$ in (1) are negligible. If the ROCOF is assumed to be equal to a constant $R$, then $\varepsilon_{p_{r}}(n)=\pi R \frac{n^{2}}{f_{s}^{2}}$. In addition, if both amplitude and phase wideband noise contributions are merged together as in (2), then (1) can be rewritten simply as

$$
x_{r}(n)=s_{r}(n, \mathbf{p})=A \cos \left(2 \pi \frac{f_{0}}{f_{s}} n+\pi R \frac{n^{2}}{f_{s}^{2}}+\phi_{r}\right)+\boldsymbol{\eta}(n),
$$

where $\mathbf{p}=\left[A, \phi_{r}, f_{0}, R\right]^{T}$ is the vector of the waveform parameters to be estimated, and $\boldsymbol{\eta}(\cdot)$ is the total wideband noise. According to the fundamentals of estimation theory [28], the CRLBs associated with the unbiased estimation of the elements of $\mathbf{p}$ are given by the diagonal elements of the inverse of the corresponding $4 \times 4$ Fischer information matrix $I(\mathbf{p})$. If the additive noise is white and normally distributed with a zero mean and variance $\sigma^{2}$ and the elements of $\mathbf{p}$ are estimated in the center of an observation interval, then the element $(i, j)$ of $I(\mathbf{p})$ is given by [28]

$$
I_{i j}(p)=\frac{1}{\sigma^{2}} \sum_{n=-\frac{N-1}{2}}^{\frac{N-1}{2}} \frac{\partial s_{r}(n, \mathbf{p})}{\partial p_{i}} \frac{\partial s_{r}(n, \mathbf{p})}{\partial p_{j}}
$$

where $p_{i}$ and $p_{j}$ denote the $i$-th and the $j$-th element of p, respectively. Note that in (A.2) $N$ is assumed to be odd. However, the case with $N$ even is very similar.

Assuming that $R$ and $N$ are small enough and that an approximately integer number of cycles is collected, the following approximate expressions hold, i.e. $\frac{1}{N^{k+1}} \sum_{n=-\frac{N-1}{2}}^{\frac{N-1}{2}} n^{k} \sin \left[2 \theta_{r}(n)\right] \cong 0$ and $\frac{1}{N^{k+1}} \sum_{n=-\frac{N-1}{2}}^{\frac{N-1}{2}} n^{k} \cos \left[2 \theta_{r}(n)\right] \cong 0$, for $k=0,1,2,3$ [28], where $\theta_{r}(n)^{2}=2 \pi \frac{f_{0}}{f_{s}} n+\pi R \frac{n^{2}}{f_{s}^{2}}+\phi_{r}$. By using these expressions, after some algebraic steps, it can be shown that the diagonal elements of $I(\mathbf{p})$ are:

$$
\begin{aligned}
& I_{11}(\mathbf{p})=\frac{1}{\sigma^{2}} \sum_{n=-\frac{N-1}{2}}^{\frac{N-1}{2}} \cos ^{2}\left[\theta_{r}(n)\right] \cong \frac{N}{2 \sigma^{2}} \\
& I_{22}(\mathbf{p})=\frac{A^{2}}{\sigma^{2}} \sum_{n=-\frac{N-1}{2}}^{\frac{N-1}{2}} \sin ^{2}\left[\theta_{r}(n)\right] \cong \frac{A^{2} N}{2 \sigma^{2}} \\
& I_{33}(\mathbf{p})=\frac{4 \pi^{2} A^{2}}{\sigma^{2} f_{s}^{2}} \sum_{n=-\frac{N-1}{2}}^{\frac{N-1}{2}} n^{2} \sin ^{2}\left[\theta_{r}(n)\right] \cong \frac{A^{2}}{2 \sigma^{2}} \frac{\pi^{2} N\left(N^{2}-1\right)}{3 f_{s}^{2}} \\
& I_{44}(\mathbf{p})=\frac{A^{2}}{\sigma^{2} f_{s}^{4}} \sum_{n=-\frac{N-1}{2}}^{2} n^{4} \sin ^{2}\left[\theta_{r}(n)\right] \cong \frac{A^{2}}{2 \sigma^{2}} \frac{\pi^{2} N\left(3 N^{2}-7\right)\left(N^{2}-1\right)}{240 f_{s}^{4}}
\end{aligned}
$$

Similarly, the non-diagonal elements of the Fischer information matrix are: $I_{12}(\mathbf{p})=I_{21}(\mathbf{p}) \cong 0, I_{13}(\mathbf{p})=I_{31}(\mathbf{p}) \cong 0, I_{41}(\mathbf{p})=$ $I_{14}(\mathbf{p}) \cong 0, I_{23}(\mathbf{p})=I_{23}(\mathbf{p}), I_{34}(\mathbf{p})=I_{43}(\mathbf{p}) \cong 0$, and

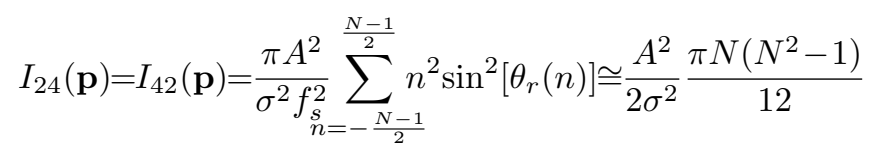

By replacing $S N R=\frac{A^{2}}{2 \sigma^{2}}$ into all elements of $I(\mathbf{p})$ and by inverting the Fischer matrix, it finally results that the CRLBs associated with any unbiased estimator of amplitude, phase, frequency and ROCOF are given respectively by

$$
\begin{aligned}
C R_{A} & \cong \frac{A^{2}}{S N R \cdot N} \\
C R_{\theta} & \cong \frac{1}{4} \frac{1}{S N R \cdot N} \frac{9 N^{2}-21}{N^{2}-4} \\
C R_{f} & \cong \frac{3 f_{s}^{2}}{\pi^{2}} \frac{1}{S N R} \frac{1}{N\left(N^{2}-1\right)} \\
C R_{R} & \cong \frac{180 f_{s}^{4}}{\pi^{2}} \frac{1}{S N R} \frac{1}{N\left(N^{2}-1\right)\left(N^{2}-4\right)} .
\end{aligned}
$$

Note that, in practice, $N$ is (at least) in the order of some tens. Thus, (A.3)-(A.6) can be approximately rewritten as (3)-(6). 


\section{APPENDIX B \\ DERIVATION OF EXPRESSION (10)}

Let $e_{A_{r}}=\hat{A}_{r}-A_{r}$ and $e_{\theta_{r}}=\hat{\theta}_{r}-\theta_{r}$ be the amplitude and phase estimation errors in the $r$-th observation interval. The probability density functions of both $\frac{e_{A_{r}}}{A_{r}}$ and $e_{\theta_{r}}$ can be reasonably assumed to be Gaussian because of the central limit theorem. If synchrophasor amplitude, phase, frequency and ROCOF are estimated using a single data record and the estimators of waveform amplitude and phase are unbiased and efficient, then $\frac{e_{A_{r}}}{A_{r}}$ and $e_{\theta_{r}}$ exhibit zero mean and variance given by (A.3) and (A.4), respectively. As a result, the scaled phase estimation error sequence $e_{\theta_{r}}^{\prime}=\frac{2}{3} e_{\theta_{r}}$ also exhibits a normal distribution with zero mean and variance $\frac{C R_{A}}{A^{2}}=\frac{4}{9} C R_{\theta}$. Thus, from the definition of TVE, it follows that if $\left|e_{\theta_{r}}\right|<<1$, we have that

$$
\begin{aligned}
T V E_{r} & =\sqrt{\frac{e_{A_{r}}^{2}}{A_{r}^{2}}+4\left(1+\frac{e_{A_{r}}}{A_{r}}\right) \sin \frac{e_{\theta_{r}}^{2}}{2}} \\
& \cong \sqrt{\frac{e_{A_{r}}^{2}}{A_{r}^{2}}+e_{\theta_{r}}^{2}}=\sqrt{e_{z_{r}}^{2}+\frac{5}{9} e_{\theta_{r}}^{2}}
\end{aligned}
$$

where random variable $e_{z_{r}}=\sqrt{\frac{e_{A_{r}}^{2}}{A_{r}^{2}}+e_{\theta_{r}}^{\prime 2}}$ exhibits a Rayleigh distribution with mean $\sqrt{\frac{\pi C R_{A}}{2 A^{2}}}$ and variance $\left(2-\frac{\pi}{2}\right) \frac{C R_{A}}{A^{2}}$. Indeed both $\frac{e_{A_{r}}}{A_{r}}$ and $e_{\theta_{r}}^{\prime}$ are normally distributed with zero mean and the same variance. Thus, if $E\{\cdot\}$ denotes the expectation operator, it ensues immediately that

$$
E\left\{T V E_{r}^{2}\right\} \cong E\left\{e_{z_{r}}^{2}\right\}+\frac{5}{9} E\left\{e_{\theta_{r}}^{2}\right\},
$$

and (10) finally results.

\section{APPENDIX C \\ DERIVATION OF EXPRESSION (16)}

Let $\tau_{T V E}, \tau_{F E_{M}}$ and $\tau_{R F E_{M}}$ be the most stringent $\mathrm{P}$ Class and M Class accuracy limits specified in [14], [16] and summarized in Section IV. By reversing (12), the system of inequalities (14) can be rewritten as

$$
\begin{aligned}
& S N R_{B} \geq \beta_{T V E}^{2} \frac{A_{i}}{C} \\
& S N R_{B} \geq \beta_{F E}^{2} \frac{B_{i}}{C^{3}} \\
& S N R_{B} \geq \beta_{R F E}^{2} \frac{\Gamma_{i}}{C^{5}}
\end{aligned}
$$

where subscript $i \in\{P, M\}$ denotes the PMU Class and

$$
A_{i}=\frac{13}{8} \frac{F^{2}}{\tau_{T V E_{i}}^{2}}, \quad B_{i}=\frac{3 f_{0}^{2}}{2 \pi^{2}} \frac{F^{2}}{\tau_{F E_{i}}^{2}}, \quad \Gamma_{i}=\frac{90 f_{0}^{4}}{\pi^{2}} \frac{F^{2}}{\tau_{R F E_{i}}^{2}} .
$$

By replacing the values of $f_{0}$ (i.e. $50 \mathrm{~Hz}$ or $60 \mathrm{~Hz}$ ), $\tau_{T V E_{i}}, \tau_{F E_{i}}$ and $\tau_{R F E_{i}}$ into (C.4), it can be easily found that $A_{i}<<B_{i}<<$ $\Gamma_{i}$ for both $i \in\{P, M\}$. Since it is clear from Tabs. I and II that usually $\beta_{T V E} \leq \beta_{F E} \leq \beta_{R F E}$, it is also evident that, for $C=1$, (C.3) dominates over both (C.1) and (C.2). However, as
$C$ grows, (C.3) decreases monotonically faster than (C.2) and much faster than (C.1). Eventually, all pairs of (C.1), (C.2) and (C.3) will return the same value for some value of $C$. Among the three intersection points, the one occurring for the smallest value of $C$ (for the considered values of constants $A_{i}, B_{i}, \Gamma_{i}$ ) is certainly associated with the intersection of the curves resulting from (C.2) and (C.3). Thus, the value of $C_{i}^{*}$ in (15) is obtained by equating (C.2) and (C.3). This means that inequality (C.3) dominates over (C.1) and (C.2) for $1 \leq C \leq C_{i}^{*}$. Moreover, since $S N R_{B}=S N R \cdot \frac{f_{s}}{2 f_{0}}$ where $S N R=10^{\frac{6.02 \cdot E N O B+1.76}{10}}$, by replacing these expressions and the estimated coefficient $\hat{\beta}_{R F E}$ into (C.3), (16) finally results.

\section{REFERENCES}

[1] J. Warichet, T. Sezi, and J. Maun, "Considerations about synchrophasors measurement in dynamic system conditions," International Journal of Electrical Power Energy Systems, vol. 31, no. 9, pp. 452 - 464, 2009.

[2] M. Wache and D. Murray, "Application of synchrophasor measurements for distribution networks," in Proc. IEEE Power and Energy Society General Meeting, San Diego, CA, USA, Jul. 2011, pp. 1-4.

[3] M. Akke and J. Thorp, "Sample value adjustment improves phasor estimation at off-nominal frequencies," IEEE Transactions on Power Delivery, vol. 25, no. 4, pp. 2255-2263, Oct. 2010.

[4] D. Belega and D. Petri, "Accuracy analysis of the multicycle synchrophasor estimator provided by the interpolated dft algorithm," IEEE Transactions on Instrumentation and Measurement, vol. 62, no. 5, pp. 942-953, May 2013.

[5] P. Romano and M. Paolone, "Enhanced interpolated-dft for synchrophasor estimation in FPGAs: Theory, implementation, and validation of a PMU prototype," IEEE Transactions on Instrumentation and Measurement, vol. 63, no. 12, pp. 2824-2836, Dec. 2014.

[6] M. Platas-Garza and J. de la O Serna, "Dynamic phasor and frequency estimates through maximally flat differentiators," IEEE Transactions on Instrumentation and Measurement, vol. 59, no. 7, pp. 1803-1811, Jul. 2010.

[7] - "Dynamic harmonic analysis through Taylor-Fourier transform," IEEE Transactions on Instrumentation and Measurement, vol. 60, no. 3, pp. 804-813, Mar. 2011.

[8] G. Barchi, D. Macii, and D. Petri, "Synchrophasor estimators accuracy: a comparative analysis," IEEE Transactions on Instrumentation and Measurement, vol. 62, no. 5, pp. 963-973, May 2013.

[9] P. Castello, J. Liu, C. Muscas, P. Pegoraro, F. Ponci, and A. Monti, "A fast and accurate pmu algorithm for P+M class measurement of synchrophasor and frequency," IEEE Transactions on Instrumentation and Measurement, vol. 63, no. 12, pp. 2837-2845, Dec. 2014.

[10] D. Belega, D. Fontanelli, and D. Petri, "Dynamic phasor and frequency measurements by an improved Taylor weighted least squares algorithm," IEEE Transactions on Instrumentation and Measurement, vol. 64, no. 8, pp. 2165-2178, Aug. 2015.

[11] M. Bertocco, G. Frigo, C. Narduzzi, C. Muscas, and P. Pegoraro, "Compressive sensing of a Taylor-Fourier multifrequency model for synchrophasor estimation," IEEE Transactions on Instrumentation and Measurement, vol. 64, no. 12, pp. 3274-3283, Dec. 2015.

[12] A. Roscoe, "Exploring the relative performance of frequency-tracking and fixed-filter phasor measurement unit algorithms under C37.118 test procedures, the effects of interharmonics, and initial attempts at merging P-Class response with M-Class filtering," IEEE Transactions on Instrumentation and Measurement, vol. 62, no. 8, pp. 2140-2153, Aug. 2013.

[13] A. Roscoe, I. Abdulhadi, and G. Burt, "P and M class phasor measurement unit algorithms using adaptive cascaded filters," IEEE Transactions on Power Delivery, vol. 28, no. 3, pp. 1447-1459, Jul. 2013. 
[14] IEEE Std C37.118.1-2011, IEEE Standard for Synchrophasor Measurements for Power Systems, Dec. 2011.

[15] IEEE Std C37.118.2-2011, IEEE Standard for Synchrophasor Data Transfer for Power Systems, Dec. 2011.

[16] IEEE Std C37.118.1a-2014 (Amendment to IEEE Std C37.118.1-2011), IEEE Standard for Synchrophasor Measurements for Power Systems - Amendment 1: Modification of Selected Performance Requirements, Apr. 2014.

[17] D. Petri, D. Fontanelli, and D. Macii, "A frequency-domain algorithm for dynamic synchrophasor and frequency estimation," IEEE Transactions on Instrumentation and Measurement, vol. 63, no. 10, pp. 23302340, Oct. 2014.

[18] P. Banerjee and S. Srivastava, "An effective dynamic current phasor estimator for synchrophasor measurements," IEEE Transactions on Instrumentation and Measurement, vol. 64, no. 3, pp. 625-637, Mar. 2015.

[19] G. Stenbakken and Ming Zhou, "Dynamic phasor measurement unit test system," in IEEE Power Engineering Society General Meeting, Tampa, FL, USA, Jun. 2007, pp. 1-8.

[20] G. Stenbakken, T. Nelson, M. Zhou, and V. Centeno, "Reference values for dynamic calibration of PMUs," in Proc. 41st Annual Hawaii International Conference on System Sciences, Waikoloa, HI, USA, Jan. 2008, pp. 1-6.

[21] A. von Meier, D. Culler, A. McEachern, and R. Arghandeh, "Microsynchrophasors for distribution systems," in IEEE PES Innovative Smart Grid Technologies Conference (ISGT), Washington, DC, USA, Feb. 2014, pp. 1-5.

[22] A. Borghetti, C. Nucci, M. Paolone, G. Ciappi, and A. Solari, "Synchronized phasors monitoring during the islanding maneuver of an active distribution network," IEEE Transactions on Smart Grid, vol. 2, no. 1, pp. 82-91, Mar. 2011.

[23] J. Hwang, "Reduction of noise in measurements of phasor angles by using two digital filters," IEEE Transactions on Power Delivery, vol. 29 , no. 1, pp. 52-60, Feb. 2014.

[24] M. Begovic, D. Novosel, and B. Djokic, "Issues related to the implementation of synchrophasor measurements," in Hawaii International Conference on System Sciences, Proceedings of the 41st Annual, Jan 2008, pp. 164-164.

[25] W. Dickerson, "Effect of PMU analog input section performance on frequency and rocof estimation error," in Proc. IEEE International Workshop on Applied Measurements for Power Systems (AMPS), Aachen, Germany, Sep. 2015, pp. 31-36.

[26] D. Macii, D. Fontanelli, D. Petri, and G. Barchi, "Impact of wideband noise on synchrophasor, frequency and ROCOF estimation," in Proc. IEEE International Workshop on Applied Measurements for Power Systems (AMPS), Aachen, Germany, Sep. 2015, pp. 43-48.

[27] B. Ristic and B. Boashash, "Comments on "the Cramer-Rao lower bounds for signals with constant amplitude and polynomial phase"," IEEE Transactions on Signal Processing, vol. 46, no. 6, pp. 1708-1709, Jun. 1998.

[28] S. M. Kay, Fundamentals of Statistical Signal Processing: Estimation Theory. Upper Saddle River, NJ, USA: Prentice-Hall, Inc., 1993.

[29] C. Offelli and D. Petri, "The influence of windowing on the accuracy of multifrequency signal parameter estimation," IEEE Transactions on Instrumentation and Measurement, vol. 41, no. 2, pp. 256-261, Apr 1992.

[30] D. Macii, D. Petri, and A. Zorat, "Accuracy analysis and enhancement of DFT-based synchrophasor estimators in off-nominal conditions," IEEE Transactions on Instrumentation and Measurement, vol. 61, no. 10, pp. 2653-2664, Oct. 2012.

[31] ISO/IEC Guide 98-3:2008, Uncertainty of measurement - Part 3: Guide to the expression of uncertainty in measurement (GUM:1995), Jan. 2008 . 